\title{
Infinite Locally Random Graphs
}

\author{
Pierre Charbit and Alex D. Scott
}

Abstract. Motivated by copying models of the web graph, Bonato and Janssen [Bonato and Janssen 03] introduced the following simple construction: given a graph $G$, for each vertex $x$ and each subset $X$ of its closed neighborhood, add a new vertex $y$ whose neighbors are exactly $X$. Iterating this construction yields a limit graph $\uparrow G$. Bonato and Janssen claimed that the limit graph is independent of $G$, and it is known as the infinite locally random graph. We show that this picture is incorrect: there are in fact infinitely many isomorphism classes of limit graph, and we give a classification. We also consider the inexhaustibility of these graphs.

\section{Introduction}

The Rado graph $\mathcal{R}$ is the unique graph with countably infinite vertex set such that, for any disjoint pair $X, Y$ of finite subsets of vertices, there is a vertex $z$ that is joined to every vertex in $X$ and no vertex in $Y$. If $0<p<1$ and $G$ is a random graph in $\mathcal{G}(\mathbb{N}, p)$, then with probability 1 we have $G \cong \mathcal{R}$. For this reason, the Rado graph is also known as the infinite random graph (see [Cameron 97] for a survey).

The Rado graph can be obtained deterministically by beginning with any finite (or countably infinite) graph $G$ and iterating the following construction:

[E1] For every finite subset $X$ of $V(G)$, add a vertex $y$ with neighborhood $N(y)=X$. 
Here, $N(x)=\{y \in V(G): x y \in E(G)\}$ is the neighborhood of $x$; we also write $N[x]=N(x) \cup\{x\}$ for the closed neighborhood of $x$.

Motivated by copying models of the web graph, Bonato and Janssen [Bonato and Janssen 03] (see also [Bodirsky and Bonato 05] and [Bonato and Janssen 05]) introduced the following interesting construction. For a finite graph $G$, the pure extension $\operatorname{PE}(G)$ of $G$ is obtained from $G$ by the following construction:

[E2] For every $x \in V(G)$ and every finite $X \subseteq N[x]$, add a vertex $y$ with neighborhood $N(y)=X$.

Iterating this construction, we obtain a limit graph, denoted by $\uparrow G$.

Bonato and Janssen [Bonato and Janssen 03, Theorem 3.3] claimed that $\uparrow G \cong \uparrow H$ for every pair $G, H$ of finite graphs. The (claimed) unique limit graph, which has become known [Bodirsky and Bonato 05] as the infinite locally random graph (see Proposition 2.1 for the reason for this name). As we show below, Bonato and Janssen's claim is incorrect. There are, in fact, infinitely many limit graphs $G$ (for instance, $\uparrow C_{5}, \uparrow C_{6}, \uparrow C_{7}, \ldots$ are all distinct), and we give a simple criterion that determines when $\uparrow G \cong \uparrow H$.

In the next section, we give a few simple properties of limit graphs $\uparrow G$; we prove our classification result in Section 3. Finally, in Section 4, we prove that for every finite $G, \uparrow G$ is inexhaustible, that is, $(\uparrow G) \backslash x \cong \uparrow G$ for all $x \in V(\uparrow G)$. This corrects another result from [Bonato and Janssen 03].

\section{Simple Properties of $\uparrow G$}

We begin with some notation. We shall refer to the vertices $y$ that are introduced in [E2] with neighborhoods contained in $N[x]$ as clones of $x$. Thus, a vertex of degree $d$ in $G$ has $2^{d+1}$ clones in $P E(G)$ (note that we take all subsets of the closed neighborhood $N[x]$ ), and $P E(G)$ contains $|G|$ isolated vertices, each one a clone of a different vertex from $G$. As indicated above, the iterating construction [E2] gives a sequence of graphs $G \subseteq P E(G) \subseteq P E^{2}(G) \subseteq \cdots$, where $P E^{n}(G)=P E\left(P E^{n-1}(G)\right)$; we write $\uparrow G$ for the limit of this sequence. We define the level $L(x)$ of a vertex of $\uparrow G$ to be the least integer $k$ such that it is contained in $P E^{k}(G)$ (where $L(x)=0$ for all $x \in V(G)$ ). For a finite subset $X \subseteq V(\uparrow G)$, we write $L(X)=\max _{x \in X} L(x)$. We also write $L^{(k)}(\uparrow G)$ for the vertices of level $k$ in $\uparrow G$ and $L^{(\leq k)}(\uparrow G)$ for the vertices of level $k$ or less. Note that, by the construction, $L^{(k)}(\uparrow G)$ is an independent set for every $k \geq 1$.

Given a graph $H$, a graph $G$ is locally $H$ if, for every vertex $x$ of $G$, the graph induced by the neighborhood $N(x)$ of $x$ is isomorphic to $H$. 
Bonato and Janssen note the following property of the construction defined above.

Proposition 2.I. [Bonato and Janssen 03] For every finite graph $G, \uparrow G$ is locally $\mathcal{R}$.

Proof. For every $x \in V(\uparrow G)$ and every $X$ and $Y$ finite disjoint subsets of $N(x)$, we want to find a vertex $z$ such that $z$ is adjacent to every vertex in $X$ and to none in $Y$. This is possible by the definition of $\uparrow G$ by taking a suitable vertex $z$ of level $L(X \cup Y)+1$.

Since $\mathcal{R}$ is the (unique) infinite random graph, it therefore makes sense to refer to $\uparrow G$ as an infinite locally random graph.

Corollary 2.2. Let $G$ be a finite graph. Then, $\uparrow G$ is $\aleph_{0}$-universal (that is, $\uparrow G$ contains every countable graph $H$ as an induced subgraph).

Another easy but important remark concerns the distance between vertices.

Proposition 2.3. Let $G$ be a finite graph and $x$ and $y$ two vertices of $P E^{k}(G)$, for some integer $k \geq 0$. Then, the distance between $x$ and $y$ is the same in $P E^{k}(G)$ and in $\uparrow G$.

Proof. It is sufficient to note that the pure extension construction [E2] does not change the distance between vertices.

We also note the following simple property.

Lemma 2.4. Let $G$ be a finite graph and $x$ a vertex of $\uparrow G$. Let $X$ be a finite subset of $N(x)$. Then, there exists a vertex $y$ with $L(y) \leq L(X)$ such that $X \subseteq N[y]$.

Proof. Let $x_{0}$ be a vertex of minimal level with $X \subseteq N\left[x_{0}\right]$. If $L\left(x_{0}\right) \leq L(X)$ then we can take $y=x_{0}$. Otherwise, $L\left(x_{0}\right)>L(X)$, and so $x_{0} \notin X$. But, $x_{0}$ was constructed on level $L\left(x_{0}\right)$ as the clone of some vertex $x_{1}$ with $L\left(x_{1}\right)<L\left(x_{0}\right)$. In particular, $N\left(x_{0}\right) \cap L^{\left(<L\left(x_{0}\right)\right)}(\uparrow G) \subseteq N\left[x_{1}\right]$ and so $X \subseteq N\left[x_{1}\right]$, which contradicts the minimality of $L\left(x_{0}\right)$.

For $x \in V(\uparrow G)$, we write

$$
N^{-}(x)=N(x) \cap L^{(<L(x))}(\uparrow G) .
$$

Note that $N^{-}(x)$ is the set of neighbors assigned to $x$ at time $L(x)$, when $x$ is first introduced. We say that a subgraph $G_{1}$ of $G$ is good if it is an induced 
subgraph of $G$ and, for all $x$ in $V\left(G_{1}\right), N^{-}(x) \subseteq V\left(G_{1}\right)$. Equivalently, $G_{1}$ is an induced subgraph such that $N(y) \cap V\left(G_{1}\right) \subseteq N^{-}(y)$ for all $y \in V(G) \backslash V\left(G_{1}\right)$.

In this context, Lemma 2.4 gives the following result.

Lemma 2.5. Let $G$ be a finite graph, and suppose that $H$ is a good subgraph of $\uparrow G$. Then,

$$
\forall x \in V(\uparrow G), \exists y \in V(H) \text { such that } N(x) \cap V(H) \subseteq N[y] \cap V(H) .
$$

Proof. We can assume that $x \notin V(H)$. Let $X=N(x) \cap V(H)$. Then $X \subseteq N^{-}(x)$, and by Lemma 2.4 there exists $y$ of level at most $L(X)$ with $X \subseteq N[y]$. If $L(y)=L(X)$, then, since the levels are independent sets and $X \subseteq N[y], y$ must belong to $X$ and thus to $H$. If $L(y)<L(X)$, then $y$ belongs to $H$ since $H$ is a good subgraph of $\uparrow G$.

\section{Classification}

We now investigate when $\uparrow G$ and $\uparrow H$ are isomorphic. In [Bonato and Janssen 03], the authors claim that $\uparrow G \cong \uparrow H$ for any pair of finite graphs $G$ and $H$ (this is their Theorem 3.3). Here we disprove this. Their proof seems to fail on page 209 of [Bonato and Janssen 03] at the end of the first paragraph: the equality $H_{n+1}-S \cong G_{1} \uplus \overline{K_{m}}$ does not hold because these vertices can be linked by edges. Moreover, it is not clear why this equality would imply $H-S \cong \uparrow\left(G_{1} \uplus \overline{K_{m}}\right)$ on the following line, as some vertices in $H$ can be constructed by cloning elements in $S$.

We begin with the following useful consequence of Lemma 2.5.

Theorem 3.I. Let $G$ and $H$ be finite graphs. Suppose that $G_{1} \supseteq G$ is a good subgraph of $\uparrow G$ and $H_{1} \supseteq H$ is a good subgraph of $\uparrow H$. If $G_{1} \cong H_{1}$ then $\uparrow G \cong \uparrow H$.

Proof. Let $\phi: V\left(G_{1}\right) \rightarrow V\left(H_{1}\right)$ be an isomorphism (note that, since $G_{1}$ and $H_{1}$ are good, they are induced subgraphs of $\uparrow G$ and $\uparrow H$, respectively, so this is an isomorphism between induced subgraphs). Using a classical "back and forth" argument, we extend $\phi$ one vertex at a time until, in the limit, we obtain an isomorphism between $\uparrow G$ and $\uparrow H$. Let $x \in V(\uparrow G)$ be a vertex of minimal level with $x \notin V\left(G_{1}\right)$. By Lemma 2.5, there exists $y \in V\left(G_{1}\right)$ such that $N(x) \cap$ $V\left(G_{1}\right) \subseteq N[y] \cap V\left(G_{1}\right)$. Let $z \notin V\left(H_{1}\right)$ be a clone of $\phi(y)$ with

$$
N^{-}(z)=N(z) \cap V\left(H_{1}\right)=\phi\left(N(x) \cap V\left(G_{1}\right)\right) .
$$


Such a clone is easily found: let $k=L\left(V\left(H_{1}\right)\right)$, and take the clone of $\phi(y)$ on level $k+1$ with exactly this neighborhood in $L^{(\leq k)}(\uparrow H)$. Then, $V\left(H_{1}\right) \cup\{z\}$ induces a good subgraph of $\uparrow H$, and, by minimality of $x, V\left(G_{1}\right) \cup\{x\}$ induces a good subgraph of $\uparrow G$. We can therefore extend $\phi$ by setting $\phi(x)=z$. Repeating the construction in alternate directions, we clearly obtain an isomorphism between $\uparrow G$ and $\uparrow H$.

We shall say that a vertex $x$ of a graph $G$ is inessential if there exists $y \in V(G)$, $y \neq x$, such that $N(x) \subseteq N[y]$. A graph is essential if it contains no inessential vertices. Given a graph $G$, a sequence of vertices $x_{1}, \ldots, x_{k}$ is a maximal sequence of removals if $x_{i}$ is inessential in $G \backslash\left\{x_{1}, \ldots, x_{i-1}\right\}$ for each $i$ and $G \backslash\left\{x_{1}, \ldots, x_{k}\right\}$ is an essential graph.

We shall show that every maximal sequence of removals yields the same essential graph (up to isomorphism). However, we first prove a simple lemma. We say that two vertices $x$ and $y$ in a graph $G$ are equivalent if $N(x)=N(y)$ or $N[x]=N[y]$. Equivalently, $N(x) \subseteq N[y]$ and $N(y) \subseteq N[x]$. Clearly, if $x$ and $y$ are equivalent in $G$, then $G \backslash x \cong G \backslash y$, with the obvious isomorphism given by exchanging $x$ for $y$ and leaving the other vertices fixed.

Equivalent vertices play an important role in the removal of inessential vertices.

Lemma 3.2. Suppose that $x$ and $y$ are inessential in a graph $G$, but $x$ is not inessential in $G \backslash y$. Then, $x$ and $y$ are equivalent.

Proof. Note first that, since $x$ and $y$ are inessential in $G$, there are $x^{\prime}$ and $y^{\prime}$ such that $N(x) \subseteq N\left[x^{\prime}\right]$ and $N(y) \subseteq N\left[y^{\prime}\right]$. If $x^{\prime} \neq y$ then considering the vertex $x^{\prime}$ in $G \backslash y$ shows that $x$ is inessential in $G \backslash y$, a contradiction. So, $x^{\prime}=y$, and $N(x) \subseteq N[y]$.

Now consider $y^{\prime}$. If $y^{\prime} \neq x$ then $N(x) \subseteq N[y]=\{y\} \cup N(y) \subseteq\{y\} \cup N\left[y^{\prime}\right]$ implies that $N(x) \backslash y \subseteq N\left[y^{\prime}\right]$, and so $y^{\prime}$ shows that $x$ is inessential in $G \backslash y$, a contradiction. Thus, we have $y^{\prime}=x$, and so $N(y) \subseteq N[x]$. It follows that $x$ and $y$ are equivalent.

We now show that maximal sequences of removals define a unique graph up to isomorphism.

Theorem 3.3. Suppose that $x_{1}, \ldots, x_{k}$ and $y_{1}, \ldots, y_{l}$ are two maximal sequences of removals in a finite graph $G$. Then, $G \backslash\left\{x_{1}, \ldots, x_{k}\right\} \cong G \backslash\left\{y_{1}, \ldots, y_{l}\right\}$.

Proof. We claim that we can modify the sequence $\left\{y_{1}, \ldots, y_{l}\right\}$ to obtain the sequence $\left\{x_{1}, \ldots, x_{k}\right\}$ without changing the isomorphism type of the resulting essential graph $G \backslash\left\{y_{1}, \ldots, y_{l}\right\}$. 
Suppose first that $x_{1} \notin\left\{y_{1}, \ldots, y_{l}\right\}$. Then (by maximality), $x_{1}$ is inessential in $G$ but not in $G \backslash\left\{y_{1}, \ldots, y_{l}\right\}$. Let $i$ be maximal such that $x_{1}$ is inessential in $G \backslash\left\{y_{1}, \ldots, y_{i}\right\}$. Then, by Lemma $3.2, x_{1}$ and $y_{i+1}$ are equivalent in $G \backslash\left\{y_{1}, \ldots, y_{i}\right\}$, and so we can replace $y_{i+1}$ by $x_{1}$ in the sequence $y_{1}, \ldots, y_{l}$, without affecting the isomorphism type of $G \backslash\left\{y_{1}, \ldots, y_{l}\right\}$ (the isomorphism is given by exchanging $x_{1}$ and $y_{i+1}$ ). We may therefore assume that $x_{1} \in\left\{y_{1}, \ldots, y_{l}\right\}$.

We now show that we can modify $y_{1}, \ldots, y_{l}$ so that $y_{1}=x_{1}$. Suppose that $x_{1}=y_{i+1}$ for some $i \geq 1$. If there exists some $0 \leq j<i-1$ such that $x_{1}$ is inessential in $G \backslash\left\{y_{1}, \ldots, y_{j}\right\}$ and not in $G \backslash\left\{y_{1}, \ldots, y_{j+1}\right\}$, Lemma 3.2 implies that $x_{1}$ and $y_{j+1}$ are equivalent in $G \backslash\left\{y_{1}, \ldots, y_{j}\right\}$. Therefore, we can exchange them in the sequence. We can repeat this operation as long as such an integer $j$ exists, and thus we can assume that $x_{1}=y_{i+1}$ is inessential in $G \backslash\left\{y_{1}, \ldots, y_{j}\right\}$ for all $j \leq i$. Now, if $y_{i}$ is not inessential in $G \backslash\left\{y_{1}, \ldots, y_{i-1}, x_{1}\right\}$, then (since it is inessential in $\left.G \backslash\left\{y_{1}, \ldots, y_{i-1}\right\}\right)$ Lemma 3.2 shows that $x_{1}$ and $y_{i}$ are equivalent in $G \backslash\left\{y_{1}, \ldots, y_{i-1}\right\}$. It is clear that we may therefore exchange $y_{i}$ and $y_{i+1}=x_{1}$ in the sequence $y_{1}, \ldots, y_{l}$. Repeating this argument, we move $x_{1}$ forward in the sequence $y_{1}, \ldots, y_{l}$ until $x_{1}=y_{1}$.

Finally, if $x_{1}=y_{1}$, we can work instead with the graph $G \backslash x_{1}$ and the sequences $x_{2}, \ldots, x_{k}$ and $y_{2}, \ldots, y_{l}$, continuing until one (and hence both) of the sequences is exhausted.

We shall denote the (isomorphism type of the) subgraph of $G$ obtained by deleting a maximal sequence of removals by $\downarrow G$. For instance, $\downarrow K_{n}=\downarrow C_{4}=K_{1}$, but $\downarrow C_{k}=C_{k}$ for all $k \geq 5$.

We next show that inessential vertices have no effect on limit graphs.

Corollary 3.4. Let $G$ be a finite graph and $x$ an inessential vertex of $G$. Then, $\uparrow G \cong \uparrow(G \backslash x)$.

Proof. Let $H=G \backslash x$. Since $x$ is inessential, there exists $y$ in $G$ such that $N(x) \subseteq N[y]$ in $G$. In $\uparrow H, y$ has a clone $x^{\prime}$ such that $N^{-}\left(x^{\prime}\right)=N(x) \cap V(G)$. Clearly, $G_{1}=G$ is a good subgraph of $\uparrow G$, and $V(H) \cup\left\{x^{\prime}\right\}$ induces a good subgraph $H_{1}$ of $\uparrow H$. Thus, it suffices to apply Theorem 3.1 to $G_{1}$ and $H_{1}$.

Corollary 3.4 implies the following theorem.

Theorem 3.5. Let $G$ be a finite graph. Then, $\uparrow G \cong \uparrow(\downarrow G)$.

If $H$ is an induced subgraph of $\uparrow G$, then we define two kinds of transformations on this subgraph, called reductions. 
(i) Delete an inessential vertex of $H$.

(ii) For a pair of vertices $x \in V(H)$ and $y \notin V(H)$ with $N(x) \cap V(H) \subseteq$ $N(y) \cap V(H)$, replace $H$ by the subgraph of $\uparrow G$ induced by $(V(H) \backslash x) \cup\{y\}$.

Lemma 3.6. If $H$ is a finite induced subgraph of $\uparrow G$, it is possible to apply a sequence of reductions to transform $H$ into a subgraph of $G$.

Proof. Define the weight $w\left(H^{\prime}\right)$ of an induced subgraph of $\uparrow G$ as

$$
w\left(H^{\prime}\right)=\sum_{v \in V\left(H^{\prime}\right)} L(v) .
$$

If $w(H)=0$, then $H$ is a subgraph of $G$. If $w(H)>0$, then we look for a reduction that decreases the weight or the number of vertices. If $H$ contains an inessential vertex, then delete it (this can occur at most $|H|-1$ times). Otherwise, let $x \in V(H)$ be a vertex of the highest level. Then, $N(x) \cap V(H)=$ $N(x) \cap V(H) \cap L^{(<L(x))}(\uparrow G)$, as $L^{(L(x))}(\uparrow G)$ is an independent set. Since $x$ was built at level $L(x)$ as the clone of some vertex $y$ that satisfies $N(x) \cap V(H) \cap$ $L^{(<L(x))}(\uparrow G) \subseteq N[y] \cap V(H)$ and $L(y)<L(x)$, we can replace $x$ by $y$ to obtain $H^{\prime}$ with $w\left(H^{\prime}\right)<w(H)$. Repeating this process, we eventually obtain an induced subgraph of $\uparrow G$ with weight 0 that, as already noted, is a subgraph of $G$.

We are now ready to prove our main result.

Theorem 3.7. Let $G$ and $H$ be finite graphs. Then, $\uparrow G \cong \uparrow H \Longleftrightarrow \downarrow G \cong \downarrow H$.

Proof. By Theorem 3.5, we may assume that $G$ and $H$ do not contain any inessential vertices, that is, $\downarrow G=G$ and $\downarrow H=H$. Suppose that $\uparrow G \cong \uparrow H$, and fix an isomorphism.

Let $\{1,2, \ldots, n\}$ be the vertices of $G$. We partition the vertices of $\uparrow G$ into $n$ classes in the following way. For $i=1, \ldots, n$, let $A_{i, 0}=\{i\}$, and for $j \geq 1$, let $A_{i, j}$ be the vertices of $\uparrow G$ that are clones of vertices in $A_{i, j-1}$. We then define $A_{i}=\bigcup_{j=0}^{\infty} A_{i, j}$. Thus, $A_{i}$ is the smallest set of vertices containing $i$ and closed under taking clones. It is easy to see that, for $i \neq k$, there is an edge between class $A_{i}$ and $A_{k}$ if and only if there is an edge between $i$ and $k$ (since creating a clone cannot create adjacencies between a new pair of classes). We shall say that edges between classes respect $G$.

Now, consider an isomorphic embedding $\phi$ of $G$ into $\uparrow G$. We say that $\phi$ is good if $\phi(i) \in A_{i}$ for every $i \in V(G)$. Suppose that $\phi$ is good, and let $G^{\prime}$ be the image of $G$ under $\phi$. If we apply a type (ii) reduction to some vertex of $G^{\prime}$, say $v_{i}:=\phi(i)$, 
then it is replaced by a vertex $x$ such that $N(x) \cap V\left(G^{\prime}\right) \supseteq N\left(v_{i}\right) \cap V\left(G^{\prime}\right)$. Let $A_{j}$ be the class containing $x$. Since $\phi$ is good, there is an edge between $A_{j}$ and $A_{k}$ whenever $k \in N(i)$. Since edges between classes respect $G$, this implies $N[j] \supseteq N(i)$. But, since we assumed that $G$ contains no inessential vertices, this is possible only if $i=j$. Indeed, $N(x) \cap V\left(G^{\prime}\right)=N\left(v_{i}\right) \cap V\left(G^{\prime}\right)$, or else we would introduce edges between new pairs of classes. It follows that we obtain a good embedding $\phi^{\prime}$ of $G$ by setting $\phi^{\prime}(i)=x$ and $\phi^{\prime}(j)=\phi(j)$ otherwise. This remains true for any sequence of reductions starting from a good embedding. In particular, any sequence of reductions starting from $G$ produces an induced copy of $G$ (note that reductions of type (i) are not possible at any stage).

By Lemma 3.6, any induced subgraph of $\uparrow H$ isomorphic to $G$ can be reduced to a subgraph of $H$. It follows that $G$ must be isomorphic to a subgraph of $H$. Arguing similarly the other way round, we see that $H$ is isomorphic to a subgraph of $G$, and so $G \cong H$.

Now it is clear that $\uparrow G$ is not independent of $G$ : it suffices to consider two circuits of different length (larger than four). In fact, Theorem 3.7 immediately gives the following classification of possible limit graphs.

Corollary 3.8. The isomorphism classes of limit graphs $\uparrow G$ of finite graphs $G$ are in bijective correspondence with the class of essential finite graphs.

\section{Inexhaustibility}

A graph $G$ is inexhaustible if $G \backslash x \cong G$ for every vertex $x \in V(G)$. For instance, the infinite complete graph $K_{\omega}$ and its complement are trivially inexhaustible; the Rado graph $\mathcal{R}$ is also inexhaustible. On the other hand, the infinite twoway path is not inexhaustible, as deleting any vertex increases the number of components. For results on inexhaustible graphs, see [Pouzet 81, El-Zahar and Sauer 91, Bonato and Delić 04].

Bonato and Janssen [Bonato and Janssen 03] consider the inexhaustibility of infinite graphs satisfying various properties and claim a rather general result. Let us define define two properties of (infinite) graphs as follows.

Definition 4.I. (Property A.) We say that a graph $G$ has Property $A$ if it satisfies the following condition: for every vertex $x$ of $G$, every finite $X \subseteq N[x]$, and every finite $Y \subseteq V(G) \backslash X$, there is a vertex $z \notin X \cup Y$ such that $X \subseteq N(z)$ and $Y \cap N(z)=\emptyset$. 
Definition 4.2. (Property B.) We say that a graph $G$ has Property $B$ if it satisfies the following: for every vertex $x$ of $G$, every finite $X \subseteq N(x)$, and every finite $Y \subseteq V(G) \backslash X$, there is a vertex $z \notin X \cup Y$ such that $X \subseteq N(z)$ and $Y \cap N(z)=\emptyset$.

Note that the only difference between Property A and Property B is that Property A is concerned with closed neighborhoods, while Property B is only concerned with neighborhoods. Clearly Property A implies Property B; furthermore, for any finite $G$, it is clear from the constructive step [E2] that $\uparrow G$ has Property A (and therefore Property B).

Bonato and Janssen [Bonato and Janssen 03, Theorem 4.1] claim that every graph with Property B is inexhaustible. However, there is a simple counterexample to this assertion: let $G$ be the Rado graph $\mathcal{R}$ with an additional isolated vertex $x$. Since the Rado graph is connected, and $G$ is not, it is clear that $G \backslash x \neq G$. (The proof of Bonato and Janssen in [Bonato and Janssen 03] appears to fail with the definition of their sets $S_{i}$.)

In fact, even the stronger Property A does not imply that a graph is inexhaustible. Consider the graph $G$ defined by starting from the path $x_{1} x_{2} x_{3} x_{4}$ of length 3 and then alternating the pure extension construction [E2] with the following step.

[E3] For every pair of vertices $\{x, y\} \neq\left\{x_{1}, x_{4}\right\}$, add a vertex $z$ with $N(z)=$ $\{x, y\}$.

Note that $x_{1}$ and $x_{4}$ are at distance 3 in the initial graph. The pure extension step [E2] does not change the distance between vertices, while [E3] does not create a path of length 2 from $x_{1}$ to $x_{4}$. Thus, $x_{1}$ and $x_{4}$ are at distance 3 in the limit graph. On the other hand, there are infinitely many paths of length 2 between any other pair of vertices. Thus, $G \backslash\left\{x_{1}, x_{4}\right\} \not G$, and so $G$ cannot be inexhaustible (if $G$ is inexhaustible, then clearly $G \backslash X \cong G$ for every finite $X \subseteq V(G))$.

On the positive side, we can show that, for any finite $G$, the limit graph $\uparrow G$ is actually inexhaustible.

Theorem 4.3. For every finite graph $G, \uparrow G$ is inexhaustible.

Proof. Let $v$ be any vertex of $\uparrow G$. We shall show that $\uparrow G \cong(\uparrow G) \backslash v$. Note that, since $\uparrow G \cong \uparrow P E^{L(v)}(G)$, we can replace $G$ by $P E^{L(v)}(G)$, and so we may assume that $v \in V(G)$.

On the first level above $G, v$ has a clone $v^{\prime}$ with $N(v) \cap G=N\left(v^{\prime}\right) \cap G$. Thus, we have an isomorphism between $G_{1}=G$ and $G_{2}=G \backslash v \cup\left\{v^{\prime}\right\}$. It is clear 
that $G_{1}$ and $G_{2} \cup\{v\}$ are good subgraphs. We will extend this isomorphism by a "back and forth" argument.

Suppose that we are given a partial isomorphism $\phi$ between two subgraphs $G_{1}$ and $G_{2}$ of $\uparrow G$, with the following properties:

1. $G_{1}$ and $G_{2} \cup\{v\}$ are good subgraphs of $\uparrow G$.

2. $V(G) \subseteq V\left(G_{1}\right), V(G) \backslash v \subseteq V\left(G_{2}\right)$, and $v \notin V\left(G_{2}\right)$.

3. There is a vertex $\tilde{v} \in V\left(G_{2}\right)$ such that $N(v) \cap V\left(G_{2}\right) \subseteq N(\tilde{v}) \cap V\left(G_{2}\right)$.

The vertex $\tilde{v}$ (in the third property) will change at each step of our construction. We begin by setting $\tilde{v}=v^{\prime}$, and note that our initial $G_{1}$ and $G_{2}$ satisfy the conditions above.

Let $x \in V(\uparrow G)$ be a vertex of minimal level with $x \notin V\left(G_{1}\right)$. This property implies that $N^{-}(x) \subseteq V\left(G_{1}\right)$, and so $G_{1} \cup\{x\}$ is still a good graph. By Lemma 2.5, there exists $y \in V\left(G_{1}\right)$ such that $N(x) \cap V\left(G_{1}\right) \subseteq N[y] \cap V\left(G_{1}\right)$, and we can define $\phi(x)$ by taking a clone of $\phi(y)$ of level greater than $L\left(V\left(G_{1}\right) \cup V\left(G_{2}\right)\right)$ such that

$$
N^{-}(\phi(x))=N(\phi(x)) \cap V\left(G_{2}\right)=\phi\left(N(x) \cap V\left(G_{1}\right)\right) .
$$

This extends the isomorphism, implies that $G_{2} \cup\{\phi(x), v\}$ is still a good graph, and implies that the vertex $\tilde{v}$ still satisfies the desired property.

We now go in the opposite direction. Let $z$ be a vertex of minimal level with $z \notin V\left(G_{2}\right) \cup\{v\}$ : we attempt to define $\phi^{-1}(z)$.

We distinguish two cases:

- $z v \notin E(\uparrow G)$, or $z v \in E(\uparrow G)$ and $z \tilde{v} \in E(\uparrow G)$.

As before, we can apply Lemma 2.5 to get $y \in V\left(G_{2}\right) \cup\{v\}$ such that $N(z) \cap V\left(G_{2}\right) \subseteq N[y] \cap V\left(G_{2}\right)$. If $y=v$, we can instead choose $y=\tilde{v}$.

We can then define $\phi^{-1}(z)$, as done previously, to be a suitable clone of $\phi^{-1}(y)$.

- $z v \in E(\uparrow G)$ and $z \tilde{v} \notin E(\uparrow G)$.

In this case we will have to change $\tilde{v}$, because we want the condition $N(v) \cap$ $V\left(G_{2}\right) \subseteq N(\tilde{v}) \cap V\left(G_{2}\right)$ to hold after adding $z$ to $G_{2}$. Let $w$ be a clone of $v$ such that $L(w)>L\left(V\left(G_{1}\right) \cup V\left(G_{2}\right)\right)$ and $N^{-}(w)=\left(N(v) \cap V\left(G_{2}\right)\right) \cup\{z\}$. Such a vertex exists, since $z$ is a neighbor of $v$. The only reason why the subgraph induced by $V\left(G_{2}\right) \cup\{v, w\}$ might not be a good graph is the edge $z w$. We therefore extend the isomorphism to $G_{2} \cup\{z, w\}$. Since $G_{2}$ is a good graph, we can use Lemma 2.5 as before to first extend the isomorphism to $z$. Since, by minimality of $z$, the subgraph induced by $V\left(G_{2}\right) \cup\{z, v\}$ is also 
a good graph, we can use Lemma 2.5 again to extend the isomorphism to $w$. Finally, the definition of $w$ implies that $G_{2} \cup\{z, w, v\}$ is a good graph, and we can choose the new $\tilde{v}$ to be $w$, as it satisfies the desired property.

Repeating the argument gives, in the limit, an isomorphism between $\uparrow G$ and $(\uparrow G) \backslash v$.

\section{References}

[Bodirsky and Bonato 05] M. Bodirsky and A. Bonato. "The Infinite Locally Random Graph." DFG Research Center MATHEON, Preprint \#269, 2005. Available at http://www.matheon.de/research/show_preprint.asp?action=details\&serial=269.

[Bonato and Delić 04] A. Bonato and D. Delić. "On a Problem of Cameron's on Inexhaustible Graphs." Combinatorica 24 (2004), 35-51.

[Bonato and Janssen 03] A. Bonato and J. Janssen. "Infinite Limits of Copying Models of the Web Graph." Internet Mathematics 1:2 (2003), 193-213.

[Bonato and Janssen 05] A. Bonato and J. Janssen. "Infinite Limits of the Duplication Model and Graph Folding." Proceedings of EuroComb '05, DMTCS Proceedings AE (2005), 223-228.

[Cameron 97] P. J. Cameron. "The Random Graph." In The Mathematics of Paul Erdös, Vol. II, Algorithms and Combinatorics 14, edited by R. L. Graham and J. Nešetřil, pp. 333-351. Berlin: Springer, 1997.

[El-Zahar and Sauer 91] M. El-Zahar and N. W. Sauer. "Ramsey-Type Properties of Relational Structures." Discrete Math. 94 (1991), 1-10.

[Pouzet 81] M. Pouzet. "Relations Impartibles." Dissertationes Math. (Rozprawy Mat.) 193 (1981), 43 pp.

Pierre Charbit, LIAFA, Université Denis Diderot Paris VII, Case 7014, 75205 Paris Cedex 13, France (charbit@liafa.jussieu.fr)

Alex D. Scott, Mathematical Institute, University of Oxford, 24-29 St Giles', Oxford, OX1 3LB, UK (scott@maths.ox.ac.uk)

Received July 27, 2006; accepted December 27, 2006. 